\title{
African Horse Sickness Caused by Genome Reassortment and Reversion to Virulence of Live, Attenuated Vaccine Viruses, South Africa, 2004-2014
}

\author{
Camilla T. Weyer, John D. Grewar, Phillippa Burger, Esthea Rossouw, Carina Lourens, \\ Christopher Joone, Misha le Grange, Peter Coetzee, Estelle Venter, \\ Darren P. Martin, N. James MacLachlan, Alan J. Guthrie
}

\begin{abstract}
African horse sickness (AHS) is a hemorrhagic viral fever of horses. It is the only equine disease for which the World Organization for Animal Health has introduced specific guidelines for member countries seeking official recognition of disease-free status. Since 1997, South Africa has maintained an AHS controlled area; however, sporadic outbreaks of AHS have occurred in this area. We compared the whole genome sequences of $39 \mathrm{AHS}$ viruses (AHSVs) from field AHS cases to determine the source of 3 such outbreaks. Our analysis confirmed that individual outbreaks were caused by virulent revertants of AHSV type 1 live, attenuated vaccine (LAV) and reassortants with genome segments derived from AHSV types 1, 3, and 4 from a LAV used in South Africa. These findings show that despite effective protection of vaccinated horses, polyvalent LAV may, paradoxically, place susceptible horses at risk for AHS.
\end{abstract}

\footnotetext{
A frican horse sickness (AHS) is a severe, often fatal disease of equids that is caused by AHS virus (AHSV), a member of the genus Orbivirus, family Reoviridae (1). The virus is transmitted to horses by biting midges in the genus Culicoides (2). Although AHS currently occurs only in sub-Saharan Africa, various species of Culicoides midges occur throughout the entire inhabited world, warranting concern that AHSV could spread into areas that are currently free of the virus $(1,3-5)$. Furthermore, the global range of related Culicoides-transmitted

Author affiliations: University of Pretoria, Onderstepoort, South Africa (C.T. Weyer, P. Burger, C. Lourens, C. Joone, M. le Grange, P. Coetzee, E. Venter, N.J. MacLachlan, A.J. Guthrie); Western Cape Department of Agriculture, Elsenburg, South Africa (J.D. Grewar); Wits Health Consortium, Johannesburg, South Africa (E. Rossouw); University of Cape Town, Cape Town, South Africa (D.P. Martin); University of California, Davis, CA, USA (N.J. MacLachlan)
}

DOI: http://dx.doi.org/10.3201/eid2212.160718 orbiviruses, such as bluetongue virus, has expanded recently, probably in part as a result of climate change (6). In AHS-endemic temperate regions, such as those occurring throughout much of South Africa, the disease is most prevalent in late summer (7). Efforts to prevent the catastrophic impact of AHS began soon after the determination of its viral etiology in 1900, at which time it was only the second animal virus ever described $(8,9)$. Presently, a polyvalent, live, attenuated vaccine (LAV) against AHSV (AHSV-LAV), which is produced by Onderstepoort Biological Products (Pretoria, South Africa) and provides broad protection against all 9 AHSV types (10), is used widely in South Africa and adjacent countries. This vaccine is supplied in 2 vials, each containing different combinations of AHSV types: combination 1 is trivalent and contains types 1,3 , and 4 , whereas combination 2 is tetravalent and contains types 2, 6, 7, and 8 (10). Heterologous immunity is believed to provide protection to the $2 \mathrm{AHSV}$ types, 5 and 9, that are not included in the vaccine.

AHS is the only equine disease for which the World Organisation for Animal Health (OIE) observes official recognition status, such that OIE member countries are required to have legally enforceable AHS control measures in place and are required to immediately notify OIE of any change to their country's AHS status (11). The Western Cape Province of South Africa, at the southern tip of the African continent, has historically been free from AHS, and for this reason, a legislatively defined AHS controlled area was created there in 1997 to facilitate movement of horses from South Africa. Within this area are an AHS free zone, consisting of the Cape Town metropolis; an AHS surveillance zone surrounding the free zone; and an outermost AHS protection zone (PZ) (Figure 1) (12). Movement of equids into and between these zones is strictly controlled. Vaccination with the polyvalent AHSV-LAV in the surveillance zone and free zone is allowed only with permission 


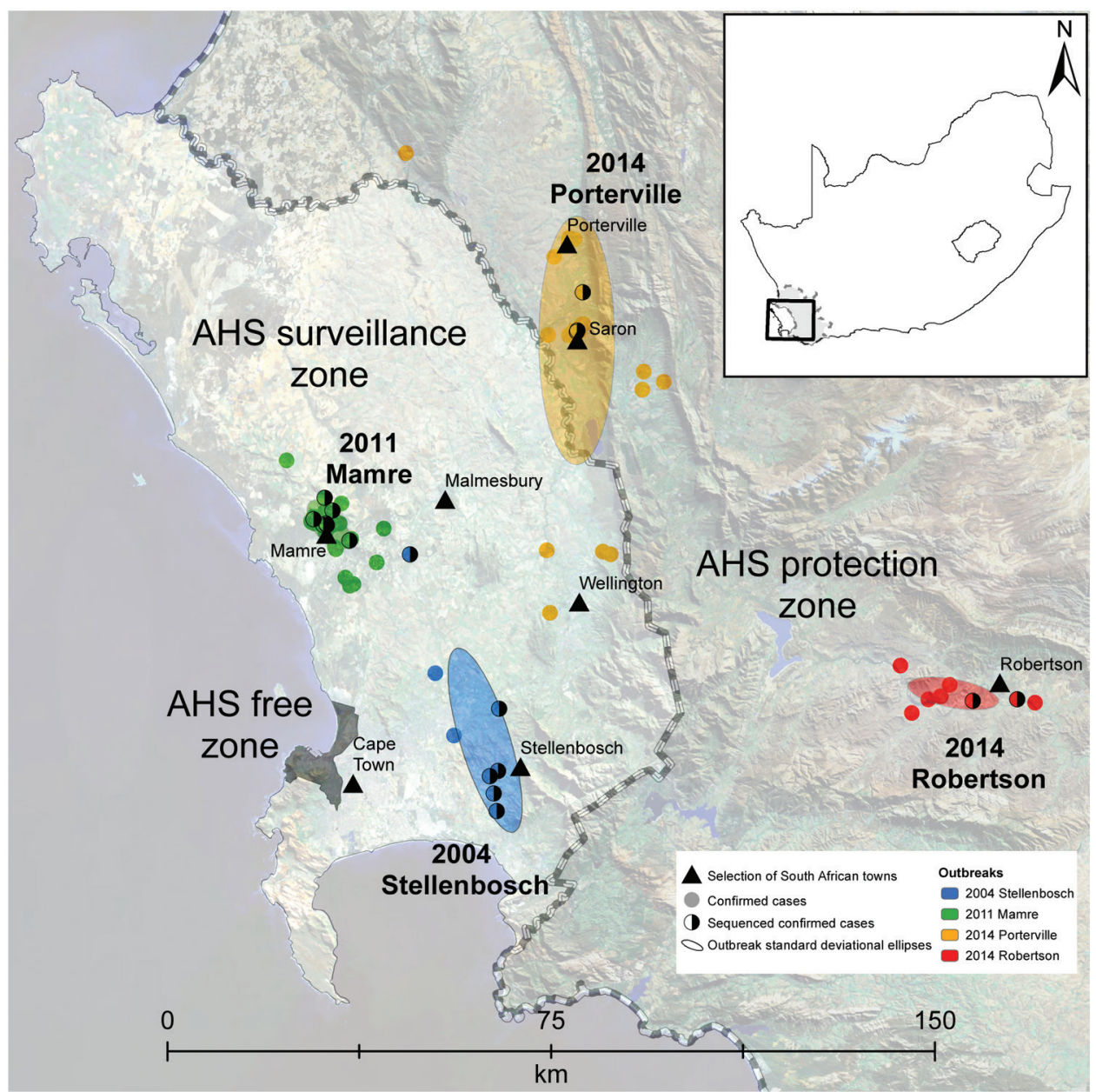

Figure 1. Locations of African horse sickness (AHS) outbreaks in Western Cape Province, South Africa, 2004-2014, including the spatial distribution of each of the AHS virus type 1 outbreaks that have occurred in the AHS controlled area since 1997. The AHS controlled area (shown in inset) is the combination of the AHS free, AHS surveillance, and AHS protection zones (also shown). Individual confirmed cases of AHS are indicated by solid dots. Half-shaded dots indicate confirmed cases for which samples were sent for sequencing (as opposed to confirmed cases that were not sequenced). The directional distribution of each outbreak is indicated by ellipses based on SD.

from the state veterinary service, and since March 2015, only during the period of low vector activity.

Since its creation in 1997, a total of 6 outbreaks of AHS in the AHS controlled area have been reported to OIE, in1999, 2004, 2006, 2011, 2013, and 2014 (13-17). Before the 2014 outbreak, these outbreaks were assumed to be caused by illegal movement of viremic animals into the controlled area, although the source was established for only 2 of these outbreaks: a type 7 virus for the 1999 outbreak in the surveillance zone and a type 5 virus for the 2006 outbreak in the PZ (Figure 1) (18,19). Because the source of the viruses responsible for the other outbreaks was never established, the goal of our study was to further characterize the epidemiology of AHSV type 1 (AHSV-1) outbreaks in the controlled area by 1) whole-genome sequencing of viruses from individual outbreaks $(2004,2011$, and 2014);2) phylogenetic comparison of these sequences with those of the polyvalent AHSV-LAV and AHSV reference strains; 3 ) analysis of outbreak viruses for genome segment reassortment; 4) analysis of single-nucleotide variants (SNVs) associated with attenuation of AHSV-LAV to determine whether vaccine-derived viruses have reverted to virulence; 5) correlation of epidemiologic and clinical findings with molecular findings; and 6) confirmation of the source of the virus strains responsible for the 2004, 2011, and 2014 outbreaks of AHS in the controlled area.

\section{Materials and Methods}

\section{Virus Isolates}

We sequenced complete genomes from 55 AHSV isolates collected during 1961-2014, including 39 field isolates of AHSV-1 from horses during the 2004 Stellenbosch (16 isolates), 2011 Mamre (7 isolates), 2014 Porterville (14 isolates), and 2014 Robertson (2 isolates) outbreaks of AHS in Western Cape Province of South Africa (Figure 1); AHSV LAV strains of types 1, 2, 3, 4, 6, 7, and 8; and Agricultural Research Council-Onderstepoort Veterinary Institute Laboratory reference strains for each of the 9 AHSV types. We included each of these virus isolates in the AHS genome sequencing Bioproject (http://www.ncbi.nlm.nih.gov/ bioproject/?term=PRJNA271179) and identified each by a unique virus strain name (online Technical Appendix 1, http:// wwwnc.cdc.gov/EID/article/22/12/16-0718-Techapp1.xlsx). 


\section{RNA Extraction, Identification, and Typing}

We isolated individual viruses of each type included in the polyvalent AHSV-LAV independently, as previously described $(20,21)$. We extracted genomic double-stranded RNA from all AHSV strains evaluated from virus-infected cells by using TRIzol reagent (Life Technologies, Johannesburg, South Africa). We identified and typed AHSV isolates by using group-specific (GS) real-time reverse transcription PCR (rRT-PCR) assays (22) and type-specific (TS) rRT-PCR assays targeting the gene encoding viral protein (VP) 2 (VP2) (23).

\section{Genome Sequencing and Assembly}

We prepared sequencing templates by using sequence-independent whole-genome RT-PCR amplification (24). We sequenced PCR amplicons on an Illumina MiSeq sequencer (Inqaba Biotechnical Industries, Pretoria, South Africa) by using the Nextera XT DNA sample preparation kit and 300-bp paired-end V3 Illumina chemistry. We analyzed Illumina sequence reads by using Geneious version 9 (http:// www.geneious.com) (25). We used a combination of de novo assembly followed by mapping to obtain the fulllength consensus genome sequences of each virus strain.

\section{Phylogenetic Analysis}

We aligned sequences of the concatenated whole virus genomes and individual genome segments by using MAFFT (http://mafft.cbrc.jp/alignment/software) (26) implemented within Geneious version 9 (25). We then used the Smart Model Selection program included in PhyML version 3 (http://www.atgc-montpellier.fr/phyml) (27) to identify the evolutionary models that best fit the individual sequence datasets by applying the corrected Akaike information criterion. We used the parameters from these models to construct maximum-likelihood trees by using PhyML version 3 (27) implemented within Geneious version 9 (25) with 1,000 bootstrap replicates to estimate branch support.

\section{Genotype Group Analysis}

We used RAMI (http://mbio-serv2.mbioekol.lu.se/rami. $\mathrm{html}$ ) to analyze the concatenated whole genome sequence maximum-likelihood tree to genetically (and not evolutionarily) classify the sequences into genotype groups based on patristic distances (28). We ran RAMI with the patristic distance threshold set to 0.000459 , enabling us to differentiate between genome sequences that differed from one another by as few as $16 \mathrm{nt}$ variants.

\section{Reassortment Analysis}

We used Recombination Detection Program (RDP) version 4.63 (29) with default settings, except that we invoked the "scan for reassortment and recombination" setting to identify any reassortment between the gene segments of LAV strains of AHSV types 1, 3, and 4 and the 39 field isolate strains included in this study. We considered reassortment events detected by any of the 8 different recombination detection methods implemented in RDP (RDP, MAXCHI, and GENECONV methods in primary scanning mode and the BURT, Bootscan, CHIMAERA, SisScan, and 3SEQ methods in secondary scanning mode, each with a Bonferroni corrected $\mathrm{p}$ value cutoff of 0.05 ) to represent evidence of reassortment.

\section{Nonsynonymous Single Nucleotide Variants}

We aligned consensus concatenated whole virus genomes from 2 AHSV-1 laboratory strains (1/Lab/ZAF/62/OVIHS29/62 and 1/Lab/ZAF/98/OBP-116) and the 39 field isolate strains by using MAFFT (26) and analyzed them by using the find variations/SNPs function in Geneious (25) with the find nonsynonymous polymorphisms only option enabled. We then compared the nonsynonymous SNVs in these sequences with the nonsynonymous SNVs previously associated with attenuation of AHSV-1 (24).

\section{Results}

\section{Phylogenetic and Genotype Group Analysis}

We used concatenated genome segments of 55 AHSV genomes to construct a maximum-likelihood phylogenetic tree incorporating best-fit substitution models (online Technical Appendix 2 Table 1, http://wwwnc.cdc.gov/ EID/article/22/12/16-0718-Techapp2.pdf) to infer degrees of genetic relatedness (Figure 2). Genotype group analysis of patristic distances inferred from this maximum-likelihood tree by using RAMI (28) indicated that the AHSV strains isolated during the 2004, 2011, and 2014 outbreaks of AHS in the controlled area segregate into 3, 1, and 2 unique groups, respectively. Specifically, the groups were 1a, 1b, and 1c for the 2004 outbreak; 2 for the 2011 outbreak; $3 \mathrm{a}$ for the 2014 Porterville outbreak; and $3 \mathrm{~b}$ for the 2014 Robertson outbreak (online Technical Appendix 2 Table 2). For the 2004 outbreak viruses, genotype group 1a includes 4 viruses that group closely with the AHSV-1LAV strain, 1/Lab/ZAF/98/OBP-116; genotype group $1 \mathrm{~b}$ includes 11 viruses that are also closely related to $1 / \mathrm{Lab} /$ ZAF/98/OBP-116; and genotype group 1c includes a single virus that segregates between 1/Lab/ZAF/98/OBP-116 and 4/Lab/ZAF/98/OBP-116. The Mamre outbreak viruses in genotype group 2 consist of 7 viruses that are all closely related to $1 / \mathrm{Lab} / \mathrm{ZAF} / 98 / \mathrm{OBP}-116$. For the 2014 outbreak viruses, genotype group $3 \mathrm{a}$ includes 14 viruses that were all isolated from AHSV-infected horses in the Porterville area and genotype group $3 \mathrm{~b}$ includes 2 viruses that were isolated from AHSV-infected horses in the Robertson area, with both groups of viruses being closely related to $1 / \mathrm{Lab} /$ ZAF/98/OBP-116. 
Table 1. Attenuation-associated nonsynonymous SNVs of consensus sequences of genome segments of AHSV-1 viruses from 4 AHS outbreaks in the AHS controlled area of Western Cape Province, South Africa, 2004-2014, and reference strains*

\begin{tabular}{|c|c|c|c|c|c|c|c|c|}
\hline \multirow[b]{2}{*}{ Abbreviated strain name } & \multicolumn{7}{|c|}{ Genome segment and amino acid position } & \multirow{2}{*}{$\begin{array}{l}\text { Genotype } \\
\text { group }\end{array}$} \\
\hline & VP2 357 & VP3 232 & VP5 422 & VP5 434 & VP6 81 & VP6 169 & NS3 201 & \\
\hline 1/E.cab-tc/ZAF/62/OVI-HS29/62 & $\mathrm{N}$ & $\mathrm{Y}$ & $\mathrm{S}$ & $\mathrm{T}$ & $\mathrm{A}$ & $\mathrm{R}$ & $\mathrm{M}$ & \\
\hline 1/Lab/ZAF/98/OBP-116† & $\mathrm{K}$ & $\mathrm{H}$ & $\mathrm{N}$ & I & V & Q & $\mathrm{K}$ & \\
\hline 1/E.cab-tc/ZAF/04/Elb-E040019 & $\ddagger$ & & & $T \S$ & $A$ & $\mathrm{R}$ & $\mathrm{E}$ & $1 a$ \\
\hline 1/E.cab-tc/ZAF/04/Elb-E040020 & & & & $T$ & A & $\mathrm{R}$ & $\bar{E}$ & $1 a$ \\
\hline 1/E.cab-tc/ZAF/04/Elb-E040021 & & & & $\mathrm{T}$ & A & $\mathrm{R}$ & $\mathrm{E}$ & $1 a$ \\
\hline 1/E.cab-tc/ZAF/04/Dkt-E040029 & & & & $\mathrm{T}$ & A & $\mathrm{R}$ & $\bar{E}$ & $1 a$ \\
\hline 1/E.cab-tc/ZAF/04/Tgd-E040031 & $\mathrm{N}$ & & & $\mathrm{T}$ & A & $\mathrm{R}$ & $\bar{\pi}$ & $1 \mathrm{~b}$ \\
\hline 1/E.cab-tc/ZAF/04/Elb-E040034 & $\mathrm{N}$ & & & $\mathrm{T}$ & A & $\mathrm{R}$ & П" & $1 \mathrm{~b}$ \\
\hline 1/E.cab-tc/ZAF/04/Tgd-E040039 & $\mathrm{N}$ & & & $\mathrm{T}$ & A & $\mathrm{R}$ & П" & $1 \mathrm{~b}$ \\
\hline 1/E.cab-tc/ZAF/04/Avt-E040043 & $\mathrm{N}$ & & & $\mathrm{T}$ & $A$ & $\mathrm{R}$ & I" & $1 \mathrm{~b}$ \\
\hline 1/E.cab-tc/ZAF/04/Avt-E040048 & $\mathrm{N}$ & & & $\mathrm{T}$ & A & $\mathrm{R}$ & I" & $1 \mathrm{~b}$ \\
\hline 1/E.cab-tc/ZAF/04/Vdm-E040062 & $\mathrm{N}$ & & & $\mathrm{T}$ & A & $\mathrm{R}$ & I" & $1 \mathrm{~b}$ \\
\hline 1/E.cab-tc/ZAF/04/Tgd-E040064 & $\mathrm{N}$ & & & $\mathrm{T}$ & A & $\mathrm{R}$ & I" & $1 \mathrm{~b}$ \\
\hline 1/E cab-tc/ZAF/04/Ndm-E040065 & $\mathrm{N}$ & & & $T$ & A & & II & $1 \mathrm{~b}$ \\
\hline 1/E cab-tc/ZAF/04/Avt-E040066 & $\mathrm{N}$ & & & $T$ & A & $\mathrm{R}$ & II & $1 \mathrm{~b}$ \\
\hline 1/E.cab-tc/ZAF/04/Avt-E040081 & $\mathrm{N}$ & & & $\mathrm{T}$ & A & $\mathrm{R}$ & I" & $1 \mathrm{~b}$ \\
\hline 1/E.cab-tc/ZAF/04/Kbk-E040086 & $\mathrm{N}$ & & & $\mathrm{T}$ & A & $\mathrm{R}$ & I" & $1 \mathrm{~b}$ \\
\hline 1/E.cab-tc/ZAF/04/Avt-E040061 & $\mathrm{N}$ & & I & I & A & $\mathrm{R}$ & I" & $1 \mathrm{c}$ \\
\hline 1/E cab-tc/ZAF/11/Mre-E110143 1 & & & & $T$ & A & $\mathrm{R}$ & $\mathrm{N}$ & 2 \\
\hline 1/E.cab-tc/ZAF/11/Mre-E110180_WC44 & & & & $\mathrm{T}$ & A & $\mathrm{R}$ & $\mathrm{N}$ & 2 \\
\hline 1/E.cab-tc/ZAF/11/Mre-E110180 WC61 & & & & $\mathrm{T}$ & A & $\mathrm{R}$ & $\mathrm{N}$ & 2 \\
\hline 1/E.cab-tc/ZAF/11/Mre-E110180 WC165 & & & & $\mathrm{T}$ & A & $\mathrm{R}$ & $\mathrm{N}$ & 2 \\
\hline 1/E.cab-tc/ZAF/11/Mre-E110411 1 & & & & $\mathrm{~T}$ & A & $\mathrm{R}$ & $\mathrm{N}$ & 2 \\
\hline 1/E.cab-tc/ZAF/11/Mre-E110418-1 & & & & $\mathrm{T}$ & A & $\mathrm{R}$ & $\mathrm{N}$ & 2 \\
\hline 1/E.cab-tc/ZAF/11/Mre-E110674 3 & & & & $\mathrm{~T}$ & A & $\mathrm{R}$ & $\mathrm{N}$ & 2 \\
\hline 1/E.cab-tc/ZAF/14/Ptv-E140485 WC00522 & & & & $\mathrm{T}$ & A & $\mathrm{R}$ & I & $3 a$ \\
\hline 1/E.cab-tc/ZAF/14/Ptv-E140485 WC00528 & & & & $\mathrm{T}$ & A & $\mathrm{R}$ & I" & $3 a$ \\
\hline 1/E.cab-tc/ZAF/14/Ptv-E140485 WC00533 & & & & $\mathrm{T}$ & A & $\mathrm{R}$ & II & $3 a$ \\
\hline 1/E.cab-tc/ZAF/14/Ptv-E140485 WC00544 & & & & $\mathrm{T}$ & A & $\mathrm{R}$ & I" & $3 a$ \\
\hline 1/E.cab-tc/ZAF/14/Ptv-E140485 WC00555 & & & & $\mathrm{T}$ & A & $\mathrm{R}$ & I" & $3 a$ \\
\hline 1/E.cab-tc/ZAF/14/Srn-E140526 WC00481 & & & & $\mathrm{T}$ & A & $\mathrm{R}$ & I" & $3 a$ \\
\hline 1/E.cab-tc/ZAF/14/Srn-E140526 WC00482 & & & & $\mathrm{T}$ & A & $\mathrm{R}$ & d" & $3 a$ \\
\hline 1/E.cab-tc/ZAF/14/Srn-E140526 WC00488 & & & & $\mathrm{T}$ & A & $\mathrm{R}$ & II & 3a \\
\hline 1/E.cab-tc/ZAF/14/Srn-E140526 WC00491 & & & & $\mathrm{T}$ & A & $\mathrm{R}$ & $\pi$ & $3 a$ \\
\hline 1/E.cab-tc/ZAF/14/Srn-E140526 Wc00493 & & & & $\mathrm{T}$ & A & $\mathrm{R}$ & II & $3 a$ \\
\hline 1/E.cab-tc/ZAF/14/Srn-E140526 wC00502 & & & & $\mathrm{T}$ & A & $\mathrm{R}$ & I" & $3 a$ \\
\hline 1/E.cab-tc/ZAF/14/Ptv-E140536 WC00506 & & & & $\mathrm{T}$ & A & $\mathrm{R}$ & I" & $3 a$ \\
\hline 1/E.cab-tc/ZAF/14/Ptv-E140536 WC00507 & & & & $\mathrm{T}$ & A & $\mathrm{R}$ & I" & $3 a$ \\
\hline 1/E.cab-tc/ZAF/14/Ptv-E140536 WC00508 & & & & $\mathrm{T}$ & A & $\mathrm{R}$ & I" & $3 a$ \\
\hline 1/E.cab-tc/ZAF/14/Rbn-E140702_RB00008 & & & & $\mathrm{T}$ & A & $\mathrm{R}$ & $\pi$ & $3 b$ \\
\hline 1/E.cab-tc/ZAF/14/Rbn-E140816_RB00221 & & & & $\mathrm{T}$ & A & $\mathrm{R}$ & वा & $3 b$ \\
\hline $\begin{array}{l}\text { *AHS, African horse sickness; AHSV-1, AHS virus } \\
\text { †The changes in amino acids are indicated in comr } \\
\text { viral proteins. } \\
\text { ¥Sequences that were identical to the consensus s } \\
\S \text { Sequences that differed from the consensus sequ } \\
\text { relevant amino acid. } \\
\text { TIndicates that these segments were not considere }\end{array}$ & $\begin{array}{l}\text { nce of the } v \\
\text { of the AHS }\end{array}$ & $\begin{array}{l}\text { accine-der } \\
/-1 \text { live, at }\end{array}$ & $\begin{array}{l}\text { d strain ar } \\
\text { huated vac }\end{array}$ & $\begin{array}{l}\text { single-nuc } \\
\text { accine-deri } \\
\text { indicated b } \\
\text { ne-derived }\end{array}$ & $\begin{array}{l}\text { strain } \\
\text { emp } \\
\text { in are }\end{array}$ & ted w & $\begin{array}{l}\text { potein. } \\
\text { BP-116) }\end{array}$ & relevant \\
\hline
\end{tabular}

Given that reassortment is a major feature of orbivirus evolution $(30,31)$, we further explored the evolutionary relationships between the 55 AHSV sequences by constructing separate maximum-likelihood trees for each of the VP1, VP2, VP3, VP4, VP5, VP6, VP7, nonstructural (NS) protein 1 (NS1), NS2, and NS3 encoding genome segments (online Technical Appendix 2 Figures 1-10). For the segments encoding VP2, VP3, VP6, NS1, and NS2, viruses included in genotype groups $1 \mathrm{a}, 1 \mathrm{~b}, 1 \mathrm{c}, 2,3 \mathrm{a}$, and $3 \mathrm{~b}$ all group, with high degrees of associated bootstrap support, together with the AHSV-1-LAV strain, 1/Lab/ZAF/98/ OBP-116. For the segments encoding VP1, VP4, and VP7, the viruses included in genotype groups 1a, 2, 3a, and $3 \mathrm{~b}$ also group with $1 / \mathrm{Lab} / \mathrm{ZAF} / 98 / \mathrm{OBP}-116$, whereas those in genotype groups $1 \mathrm{~b}$ and $1 \mathrm{c}$ group with the AHSV-3LAV strain, 3/Lab/ZAF/98/OBP-116. For the gene encoding VP5, viruses included in all genotype groups except 1c group with $1 / \mathrm{Lab} / \mathrm{ZAF} / 98 / \mathrm{OBP}-116$, whereas those in genotype group 1c group with the AHSV-4-LAV strain, 4/ Lab/ZAF/98/OBP-116. For genes encoding NS3, viruses included in genotype groups $1 \mathrm{a}$ and 2 group with $1 / \mathrm{Lab} /$ ZAF/98/OBP-116, whereas those included in the remaining genotype groups group with 4/Lab/ZAF/98/OBP-116. Collectively, these data confirm that all 10 gene segments of 
the viruses included in genotype groups $1 \mathrm{a}$ and 2 are probably derived from a most recent common ancestor closely resembling 1/Lab/ZAF/98/OBP-116; the viruses included in genotype groups $1 \mathrm{~b}$ and $1 \mathrm{c}$ are probably reassortants derived from parental viruses very closely resembling $1 / \mathrm{Lab} /$
ZAF/98/OBP-116, 3/Lab/ZAF/98/OBP-116, and 4/Lab/ ZAF/98/OBP-116; and viruses in genotype groups $3 \mathrm{a}$ and $3 \mathrm{~b}$ are probably reassortants derived from parental viruses very closely resembling 1/Lab/ZAF/98/OBP-116 and 4/ Lab/ZAF/98/OBP-116 (Figure 3).

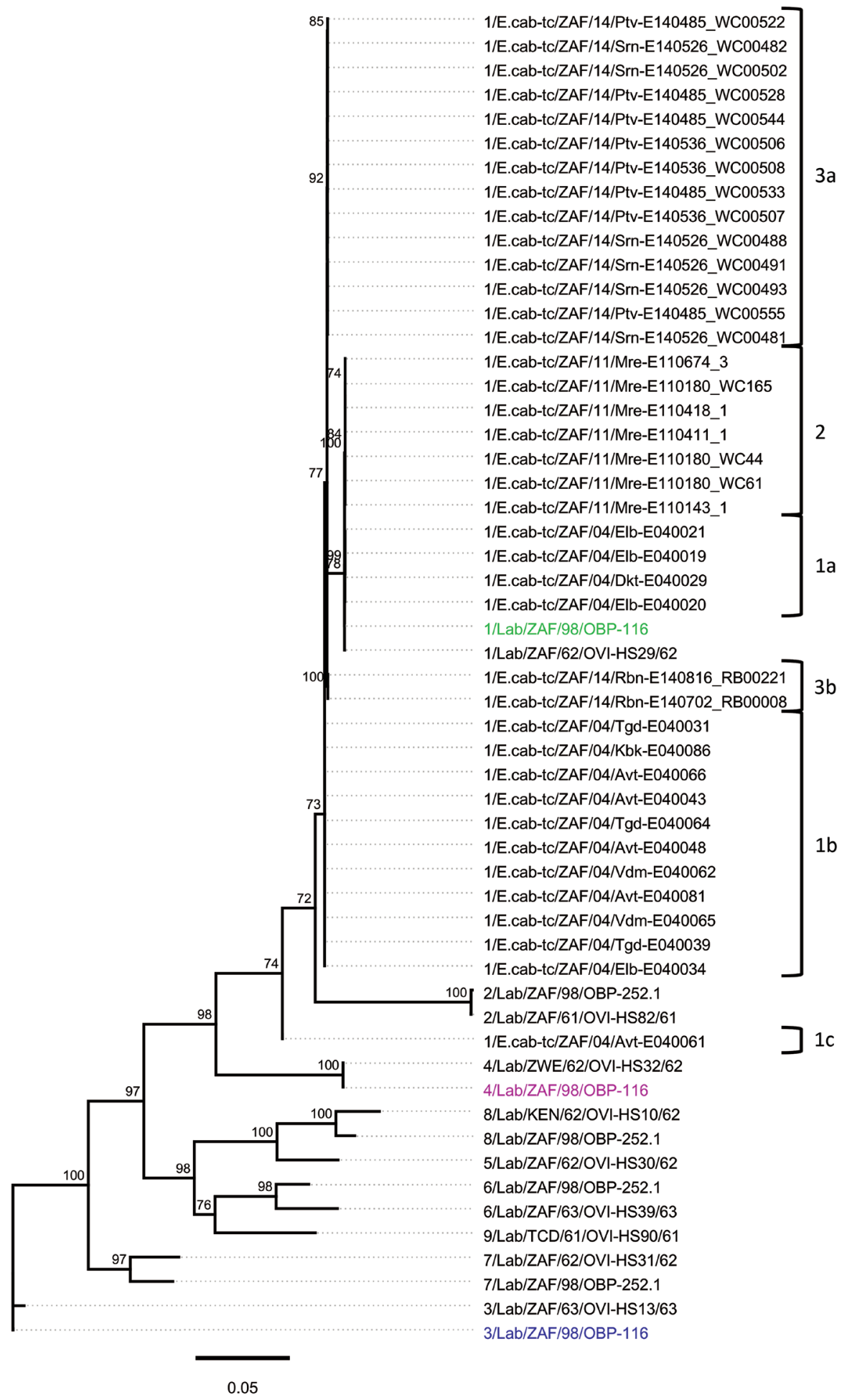

Figure 2. Whole-genome phylogeny of African horse sickness (AHS) viruses identified in AHS outbreaks in Western Cape Province, South Africa, 2004-2014. Maximum-likelihood phylogenetic tree indicating the genetic relationships of concatenated whole genome nucleotide sequences of AHS viruses from affected horses in the 2004, 2011, and 2014 outbreaks in the AHS controlled area in Western Cape Province to the AHS live, attenuated vaccine viruses and reference viruses. Green indicates vaccine-derived 1/Lab/ZAF/98/ OBP-116, blue indicates vaccinederived 3/Lab/ZAF/98/OBP-116, and red indicates vaccine-derived 4/Lab/ZAF/98/OBP-116. Branches are scaled to represent numbers of inferred nucleotide differences per site. Branches supported by full maximum-likelihood bootstrap values $>70 \%$ are indicated. Genotype groups are indicated at right. Scale bar indicates genetic distance. 
Table 2. Epidemiologic parameters for 4 outbreaks involving AHS virus type 1 in the AHS controlled area in Western Cape Province, South Africa, 2004-2014*

\begin{tabular}{|c|c|c|c|c|}
\hline Parameter† & 2004 Stellenbosch & 2011 Mamre & 2014 Porterville & 2014 Robertson \\
\hline No. confirmed cases & $23(16) \ddagger$ & $84(73) \S$ & 89 & 22 \\
\hline No. deaths & $18(16) \ddagger$ & $64(64) \S$ & 13 & 1 \\
\hline Case-fatality rate, \% & $78.3(100) \ddagger$ & $76.2(87.7) \S$ & 14.6 & 4.5 \\
\hline No. subclinical cases & 0 & $15(4) \dagger$ & 52 & 17 \\
\hline$\%$ Subclinical & 0 & $17.9(5.5) \S$ & 58.4 & 77.3 \\
\hline No. vaccinated cases & $2 / 23$ & $2 / 84$ & $35 / 89$ & $3 / 22$ \\
\hline$\%$ Vaccinated & 8.7 & 2.4 & 39.3 & 13.6 \\
\hline No. properties affected & $10(8) \ddagger$ & $47(45) \S$ & 31 & 8 \\
\hline \multicolumn{5}{|c|}{$\begin{array}{l}{ }^{*} \mathrm{AHS}, \text { African horse sickness. } \\
\dagger \text { The parameters were calculated by using the current World Organization for Animal Health (OIE) case definition. Parameters calculated by using the } \\
\text { case definitions when the outbreaks occurred are in parenthesis for the } 2004 \text { and } 2011 \text { outbreaks. } \\
\ddagger \text { An additional } 5 \text { clinical cases and } 2 \text { deaths that met the criteria of the current OIE AHS case definition were not included based on the case definition in } \\
\text { place at the time of this outbreak (13). } \\
\S A n \text { additional } 11 \text { subclinical cases that met the criteria of the current OIE AHS case definition were not included based on the case definition in place at } \\
\text { the time of this outbreak (15). }\end{array}$} \\
\hline
\end{tabular}

Explicitly testing for intrasegment recombination and reassortment by using RDP4.63 (29) yielded no evidence of intracomponent recombination in any virus but strong evidence of reassortment in genotype group $1 \mathrm{~b}, 1 \mathrm{c}, 3 \mathrm{a}$, and $3 \mathrm{~b}$ viruses (online Technical Appendix 2 Table 3). Genotype group $1 \mathrm{~b}$ viruses have 6 genome segments (encoding VP2, VP3, VP5, VP6, NS1, and NS2) derived from a virus resembling 1/Lab/ZAF/98/OBP-116; 2 segments (encoding VP1 and VP7) derived from a virus resembling 3/ $\mathrm{Lab} / \mathrm{ZAF} / 98 / \mathrm{OBP}-116$ (multiple testing corrected $\mathrm{p}=2.27$ $\times 10^{-12}$ and $1.13 \times 10^{-31}$, respectively); 1 segment (encoding NS3) derived from a virus resembling 4/Lab/ZAF/98/ OBP-116 $\left(\mathrm{p}=9.31 \times 10^{-240}\right)$; and 1 segment (encoding VP4) that could plausibly have been derived from either $3 / \mathrm{Lab} / \mathrm{ZAF} / 98 / \mathrm{OBP}-116$ or $1 / \mathrm{Lab} / \mathrm{ZAF} / 98 / \mathrm{OBP}-116$ $\left(p=7.66 \times 10^{-4}\right)($ Figure 4$)$. Genotype group $1 \mathrm{c}$ viruses display a reassortant pattern resembling that of group $1 \mathrm{~b}$ viruses except that the segment encoding VP5 is apparently derived from a virus resembling 4/Lab/ZAF/98/OBP-116 $\left(p=1.96 \times 10^{-216}\right)$. Genotype groups $3 a$ and $3 b$ viruses have 9 segments derived from a virus resembling 1/Lab/ZAF/98/ OBP-116 and a single segment (NS3) derived from a virus resembling 4/Lab/ZAF/98/OBP-116 $\left(p=9.31 \times 10^{-240}\right)$ (Figure 4).

Several SNVs relative to the AHSV-LAV-derived viruses 1/Lab/ZAF/98/OBP-116 and 3/Lab/ZAF/98/OBP116 are present in the NS1-encoding genes of viruses included in genotype groups $3 \mathrm{a}$ (2014 Porterville) and 3b (2014 Robertson) (online Technical Appendix 2 Table 4). Only a single nonsynonymous SNV exists between the NS1-encoding genes of 1/Lab/ZAF/98/OBP-116 and 3/ Lab/ZAF/98/OBP-116 (NS1 I264T). All viruses included in genotype group 3a (2014 Porterville) have the I amino acid variant that is present in $1 / \mathrm{Lab} / \mathrm{ZAF} / 98 / \mathrm{OBP}-116$, whereas viruses in genotype group $3 \mathrm{~b}$ (2014 Robertson) include the $\mathrm{T}$ amino acid variant present in 3/Lab/ZAF/98/ OBP-116. Viruses in the $3 \mathrm{~b}$ genotype group (2014 Robertson) include $\geq 2$ synonymous SNVs and $\geq 1$ nonsynonymous
SNV relative to $3 / \mathrm{Lab} / \mathrm{ZAF} / 98 / \mathrm{OBP}-116$, which suggests that the NS1 gene of the virus strains in genotype group $3 \mathrm{~b}$ are most probably derived from 3/Lab/ZAF/98/OBP-116, whereas those in genotype group $3 \mathrm{a}$ are more probably derived from 1/Lab/ZAF/98/OBP-116.

Seven nonsynonymous SNVs were identified between the whole genome sequences of the AHSV-1-LAV-derived virus, 1/Lab/ZAF/98/OBP-116, and its parental virus, 1/E.cab-tc/ZAF/62/OVI-HS29/62 (Table 1). SNVs are present at 4 of these 7 sites in the 4 viruses included in genotype group 1a. Intriguingly, 3 of these 4 changes are apparently reversions to the nonsynonymous SNV that is present in the virulent parental virus (I434T in VP5 and V81A and Q169R in VP6) and are therefore potentially reversion-to-virulence mutations. The 1 other SNV in the genotype group 1a viruses is site 201 in NS3, whereas in 1/Lab/ZAF/98/OBP-116 and 1/E.cab-tc/ZAF/62/OVIHS29/62, a K and an M, respectively, are at this site, and in the group 1a viruses, an $\mathrm{E}$ is at this site. In 10 of the 11 field viruses in genotype group $1 \mathrm{~b}$, nonsynonymous SNVs were also detected at 4 of the 7 sites that differentiate the attenuated 1/Lab/ZAF/98/OBP-116 virus from its virulent parent, 1/E.cab-tc/ZAF/62/OVI-HS29/62. The remaining field virus in genotype group $1 \mathrm{~b}, 1 / \mathrm{E}$.cab-tc/ ZAF/04/Vdm-E040065, includes 3 of these 4 SNVs. The I434T SNV in VP5 and the V81A and Q169R SNVs in VP6 of viruses in genotype group 1a are the same as those found in the genotype group $1 \mathrm{~b}, 2,3 \mathrm{a}$, and $3 \mathrm{~b}$ viruses. The K357N SNV in VP2 was detected only among viruses in genotype groups $1 \mathrm{~b}$ and $1 \mathrm{c}$. All the viruses included in genotype groups $1 \mathrm{~b}, 1 \mathrm{c}, 3 \mathrm{a}$, and $3 \mathrm{~b}$ are also reassortants with an NS3-encoding segment derived from a virus resembling 4/Lab/ZAF/98/OBP-116; therefore, SNVs in this component of these viruses were not considered as genuine mutationally derived SNVs.

The 1/E.cab-tc/ZAF/04/Avt-E040061 strain in genotype group 1c has nonsynonymous SNVs at 3 of the 7 loci (K357N in VP2 and V81A and Q169R in VP6) but a 
Strain

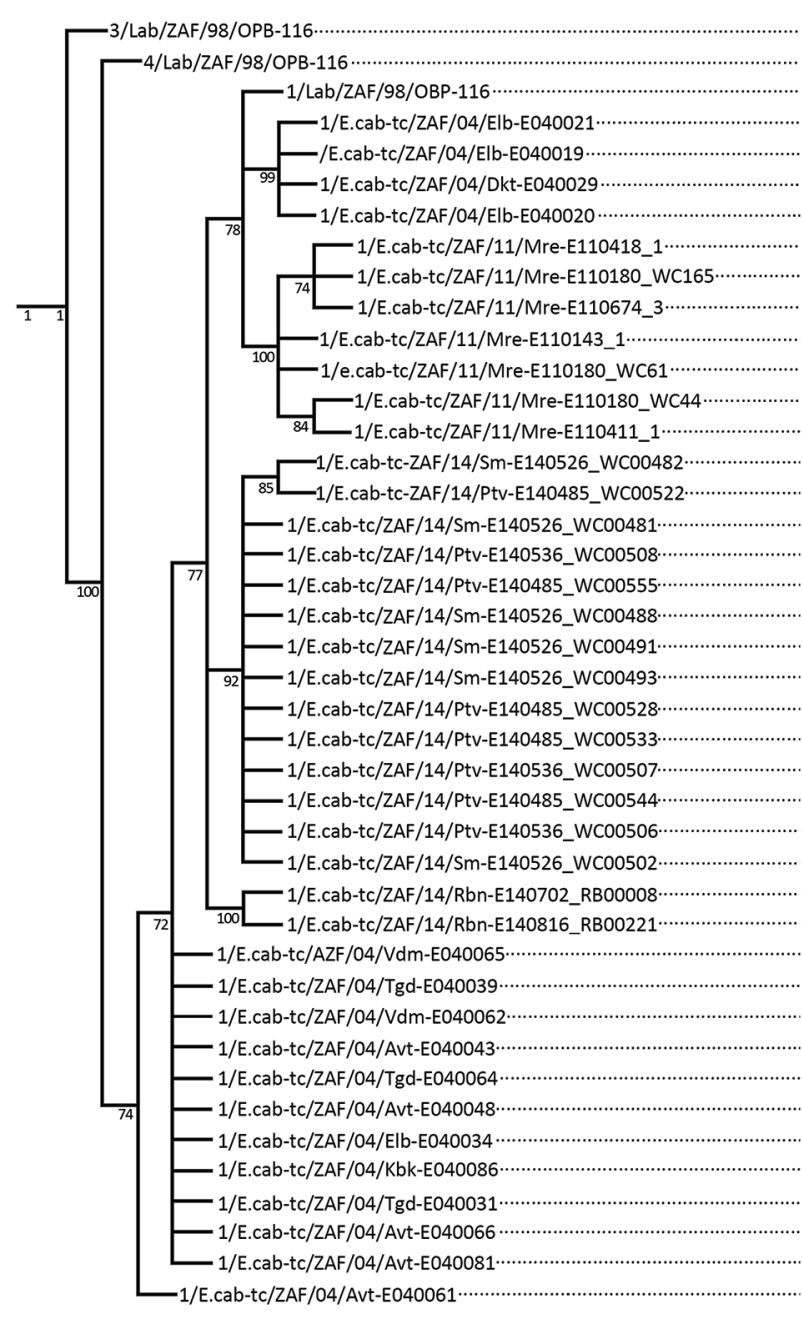

Gene

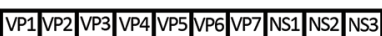

\begin{tabular}{|l|l|l|l|l|l|l|l|l|l|}
\hline 3 & 3 & 3 & 3 & 3 & 3 & 3 & 3 & 3 & 3 \\
\hline
\end{tabular}

\begin{tabular}{|l|l|l|l|l|l|l|l|l|l|}
\hline 4 & 4 & 4 & 4 & 4 & 4 & 4 & 4 & 4 & 4 \\
\hline 1 & 1 & 1 & 1 & 1 & 1 & 1 & 1 & 1 & 1 \\
\hline
\end{tabular}

\begin{tabular}{|llllllllll|}
\hline 1 & 1 & 1 & 1 & 1 & 1 & 1 & 1 & 1 & 1 \\
\hline
\end{tabular}

\begin{tabular}{|llllllllll|}
\hline 1 & 1 & 1 & 1 & 1 & 1 & 1 & 1 & 1 & 1 \\
\hline 1 & 1 & 1 & 1 & 1 & 1 & 1 & 1 & 1 & 1 \\
\hline 1 & 1 & 1 & 1 & 1 & 1 & 1 & 1 & 1 & 1 \\
$1 \mathrm{a}$
\end{tabular}

\begin{tabular}{|llllllllll|}
\hline 1 & 1 & 1 & 1 & 1 & 1 & 1 & 1 & 1 & 1 \\
\hline & 1 & 1 & 1 & 1 & 1 & 1 & 1 & 1 & 1 \\
\hline
\end{tabular}

\begin{tabular}{|l|l|l|l|l|l|l|l|l|l|}
\hline 1 & 1 & 1 & 1 & 1 & 1 & 1 & 1 & 1 & 1 \\
\hline
\end{tabular}

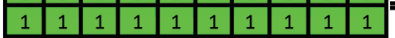

\begin{tabular}{|l|l|l|l|l|l|l|l|l|l|}
\hline 1 & 1 & 1 & 1 & 1 & 1 & 1 & 1 & 1 & 1 \\
\hline 1 & 1 & 1 & 1 & 1 & 1 & 1 & 1 & 1 & 1 \\
\hline
\end{tabular}

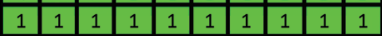

\begin{tabular}{|llllllll|l|l|}
\hline 1 & 1 & 1 & 1 & 1 & 1 & 1 & 1 & 1 & 1 \\
\hline 1 & 1 & 1 & 1 & 1 & 1 & 1 & 1 & 1 & 1 \\
\hline 1 & 1 & 1 & 1 & 1 & 1 & 1 & 1 & 1 & 1 \\
\hline
\end{tabular}

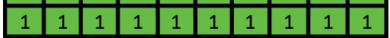

\begin{tabular}{|llllllllll|l|}
\hline 1 & 1 & 1 & 1 & 1 & 1 & 1 & 1 & 1 & 1 \\
\hline 1 & 1 & 1 & 1 & 1 & 1 & 1 & 1 & 1 & 1 \\
\hline 1 & 1 & 1 & 1 & 1 & 1 & 1 & 1 & 1 & 1 \\
\hline
\end{tabular}

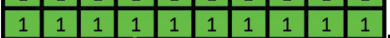

\begin{tabular}{lllll|l|l|l|l|l|}
1 & 1 & 1 & 1 & 1 & 1 & 1 & 1 & 1 & 1 \\
\hline 1 & 1 & 1 & 1 & 1 & 1 & 1 & 1 & 1 & \\
\hline & 1 & 1 & 1 & 1 & 1 & 1 & 1 & 1 & 4 \\
\hline
\end{tabular}

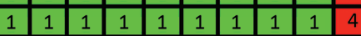

\begin{tabular}{|l|l|l|l|l|l|l|l|l|l|}
\hline 1 & 1 & 1 & 1 & 1 & 1 & 1 & 1 & 1 & 4 \\
\hline 1 & 1 & 1 & 1 & 1 & 1 & 1 & & 1 & 4 \\
\hline
\end{tabular}

\begin{tabular}{|lllllllllll}
\hline & 1 & 1 & 1 & 1 & 1 & 1 & 1 & 1 & 1 & 4 \\
\hline 1 & 1 & 1 & 1 & 1 & 1 & 1 & 1 & 1 & 4 \\
\hline & 1 & 1 & 1 & 1 & 1 & 1 & 1 & 1 & 4 \\
\hline
\end{tabular}

\begin{tabular}{|l|l|l|l|l|l|l|l|l|l|}
\hline 1 & 1 & 1 & 1 & 1 & 1 & 1 & 1 & 1 & 4 \\
\hline
\end{tabular}

\begin{tabular}{|lllllllllll}
\hline 1 & 1 & 1 & 1 & 1 & 1 & 1 & 1 & 1 & 4 \\
\hline 1 & 1 & 1 & 1 & 1 & 1 & 1 & 1 & 1 & 4 \\
\hline 1 & 1 & 1 & 1 & 1 & 1 & 1 & 1 & 1 & 4 \\
\hline
\end{tabular}

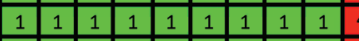

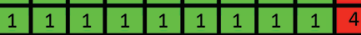

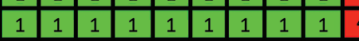

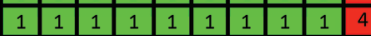

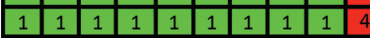

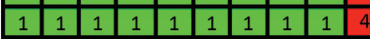

\begin{tabular}{|l|l|l|l|l|l|l|l|l|l|}
\hline 1 & 1 & 1 & 1 & 1 & 1 & 1 & 1 & 1 & \\
\hline
\end{tabular}

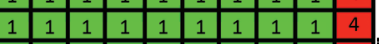

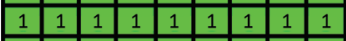

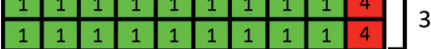

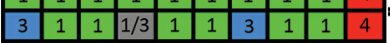

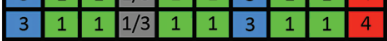

\begin{tabular}{|l|l|l|l|l|l|l|l|l|l|}
\hline 3 & 1 & 1 & $1 / 3$ & 1 & 1 & 3 & 1 & 1 & 4 \\
\hline 3 & 1 & 1 & $1 / 3$ & 1 & 1 & 3 & 1 & 1 & 4 \\
\hline
\end{tabular}

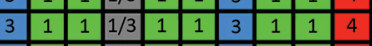

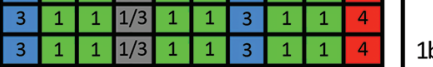

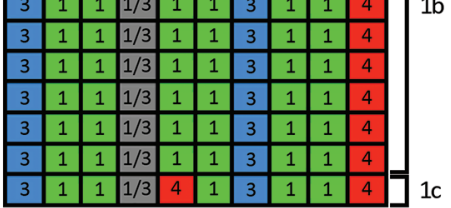

Figure 3. Cladogram and heat map of vaccine-derived African horse sickness (AHS) virus reassortants identified in AHS outbreaks in Western Cape Province, South Africa, 2004-2014. Cladogram indicates genetic relationships of concatenated AHS virus wholegenome nucleotide sequences from affected horses in the 2004, 2011, and 2014 outbreaks in the AHS controlled area in Western Cape Province. Heat map diagram summarizes the origin of the gene segments for each strain with $1 / \mathrm{Lab} / \mathrm{ZAF} / 98 /$ OBP-116 (green blocks), 3/Lab/ ZAF/98/OBP-116 (blue blocks), and 4/Lab/ZAF/98/OBP-116 (red blocks) vaccine-derived 3a strains. Gray blocks indicate that the segment could be derived from either 1/Lab/ZAF/98/OBP116 or 3/Lab/ZAF/98/OBP-116. Branches supported by full maximum-likelihood bootstrap values $>70 \%$ are indicated.

Genotype groups are indicated at right.
VP5-encoding gene apparently derived by reassortment from a virus resembling $4 / \mathrm{Lab} / \mathrm{ZAF} / 98 / \mathrm{OBP}-116$, such that the SNVs in the VP5 of this strain were also not considered to be mutationally derived.

The 7 viruses included in genotype group 2 and the 16 viruses included in genotype groups $3 \mathrm{a}$ and $3 \mathrm{~b}$ all exhibit potential reversion-to-virulence mutations at 3 of the 7 nonsynonymous SNV sites that differentiate the AHSV1-LAV virus from its virulent parent (I434T in VP5 and V81A and Q169R in VP6). Additionally, a fourth SNV (K201N in NS3) at 1 of the 7 sites differentiating the AHSV-1 LAV from its parent (which had a K and an M, respectively, at this site) is also present in the genotype group 2 viruses.

\section{Quantification of the Outbreaks}

The epidemiologic parameters of the AHS outbreaks in the controlled area in 2004, 2011, and 2014 were inferred by using the current OIE case definition for AHS (11)
(Table 2). Although the case-fatality rates (CFRs) were very high for the 2004 Stellenbosch $(78.3 \%)$ and 2011 Mamre (76.2\%) outbreaks, they were considerably lower for the 2014 Porterville (14.6\%) and Robertson (4.5\%) outbreaks. Additionally, the 2011 Mamre and 2004 Stellenbosch outbreaks were associated with the lowest vaccination rates among AHSV-infected horses $(2.7 \%$ and $8.7 \%$, respectively). Differences in the genetic constitution of the individual outbreak viruses could have been associated with the vastly different CFRs in each outbreak; however, whether these differences in CFRs are a consequence of lower virulence among the outbreak viruses or the result of existing vaccine-induced immunity in the exposed horses is unknown. Similarly, changes in the AHS case definition that only came into effect in 2008 (after the 2004 Stellenbosch outbreak) probably resulted in an underestimation of subclinical AHSV infections during that outbreak. Whereas during the Stellenbosch 2004 outbreak only clinically affected, deceased horses were classified 
Figure 4. Statistical evidence of reassortment within the genomes of African horse sickness (AHS) virus field isolates identified in outbreaks in the AHS controlled area in Western Cape Province, South Africa, 2004-2014. A hidden Markov model-based approach (BURT-HMM) was used to classify individual nucleotides within each of the 10 segments of individual AHS virus isolates into 3 different categories: $1 /$ Lab/ZAF/98/OBP-116-like (green), 3/Lab/ZAF/98/OBP116-like (blue), and 4/Lab/ ZAF/98/OBP-116-like (red). Probability supports for these classifications yielded by the BURT-HMM with the highest likelihood are plotted along the genome. Positions of segment boundaries are given in the diagram above the plots. The phylogenetic clusterings that are implied by differently colored segments in these plots are indicated below the plots. The segment indicated in gray could not be convincingly classified because it closely resembles both 1/Lab/ZAF/98/OBP-116 and 3/Lab/ZAF/98/OBP-116.

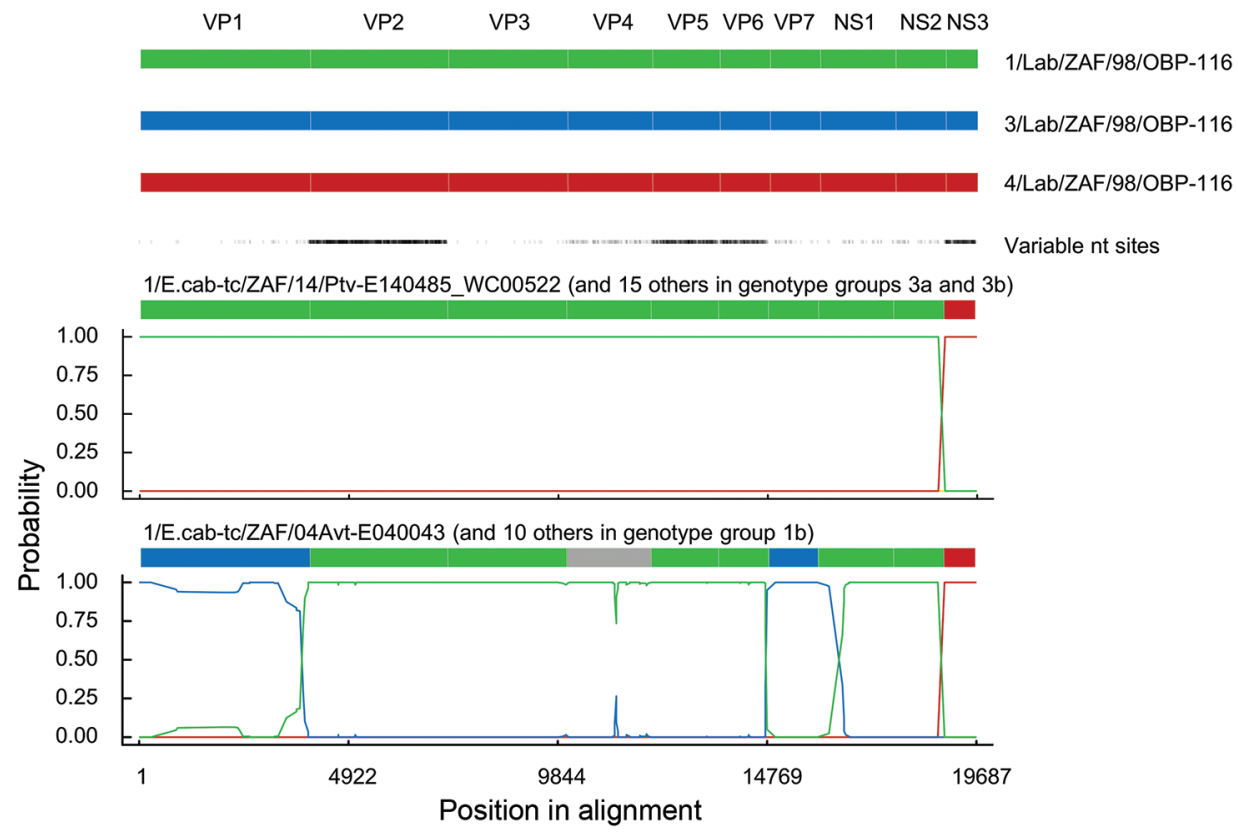

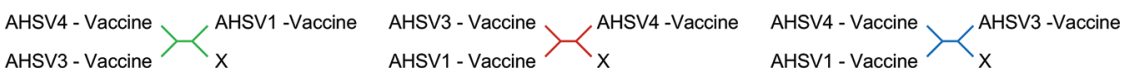

as having confirmed cases (13), major advances in AHS diagnostic testing (e.g., rRT-PCR-based methods) have occurred during the past 10 years that likely substantially increased the detection of subclinical infections by the time of the 2014 outbreaks $(15,32)$.

\section{Discussion}

Whole-genome sequences were compared from 55 field, LAV, and laboratory strains of AHSV. The field viruses were obtained from horses during outbreaks of AHS of different clinical severity (CFRs ranging from $4.5 \%$ to $78.3 \%$ ) in the AHS controlled area of South Africa during 2004, 2011, and 2014. Phylogenetic analyses confirmed that genetically distinct viruses were responsible for each outbreak and that these were all closely related to viruses contained in the trivalent AHSV-LAV (combination 1) used in South Africa. Evaluation of nonsynonymous SNVs confirmed some outbreak viruses to be revertants of the vaccine AHSV-1 strain toward the virulent parental type. Furthermore, some outbreak viruses were clearly reassortants with individual genome segments derived from multiple different virus types that are present in the trivalent vaccine preparation.

Potgieter et al. (24) hypothesized that changes in VP2 and VP5 can confer virulence or attenuation of individual AHSV strains, based upon comparisons of the consensus sequences of the genome of an attenuated AHSV-1 isolate (GenBank accession nos. FJ183364-FJ183373) and its virulent parent. Potgieter et al. (24) also proposed that virulence is related to tissue tropism because the outer capsid proteins are involved in cell entry and trigger apoptosis of host cells. Additionally, other studies have implicated NS3 as a determinant of AHSV virulence (33). The results of our study further confirm that changes in multiple VPs can affect the virulence of AHSV. Both reversion (to the virulent parental type) and novel SNVs were present in field-isolated viruses at various residue sites in VP2 (K357N in genotype group $1 \mathrm{~b}$ viruses), VP5 (I434T in all field viruses evaluated except sample 1/E. cab-tc/ZAF/04/Avt- E040061), and VP6 (V81A in all field viruses and Q169R in all field viruses except sample 1/E.cab-tc/ZAF/04/Vdm-E040065) that differentiate the attenuated AHSV-1-LAV strain from its virulent parental strain. Furthermore, SNVs present at a site in NS3 (K201E in genotype group 1a viruses and $\mathrm{K} 201 \mathrm{~N}$ in genotype group 2) are potentially associated with reversion to virulence because of the effect of NS3 on virus release, membrane permeability, and viral yield (34). However, the determinants of AHSV virulence are probably complex and multigenic $(24,34)$, which is consistent with the remarkable difference in CFRs between horses in the various outbreaks.

Given the genetic diversity of field strains of AHSV $(14,24,35)$, our analyses overwhelmingly support the 
premise that the potential reversion-to-virulence mutants and reassortants that we detected arose from viruses within the polyvalent AHSV-LAV formulation, and predominantly from AHSV-1-LAV. Although these mutants and reassortants most likely arose within vaccinated horses, the reason for the predominance of AHSV-1-LAV components in the emergent outbreak viruses is unknown. The data presented here also indicate that distinct founder events led to the expansion in Stellenbosch (2004) of viruses included in genotype groups $1 \mathrm{a}$ and $1 \mathrm{~b}$ and, similarly, that the outbreaks in 2014 in Porterville (genotype group 3a) and Robertson (genotype group $3 \mathrm{~b}$ ) also probably originated independently from the LAV and were not from the spread of the same outbreak virus.

In summary, results of this study highlight the importance of genetic characterization of circulating strains of AHSV in epidemiologic investigations of AHS outbreaks. Although, the prevailing opinion in South Africa was that illegal movement of viremic equids into the AHS controlled area was responsible for the repeated occurrences of AHS in the controlled area, this is clearly not the only cause. Our data confirm that use of polyvalent AHSV-LAV can result in the emergence and spread of virulent viruses to adjacent susceptible horses, presumably by Culicoides midge vectors that are already resident within the AHS controlled area (36). Collectively, these findings have major implications for strategies to control AHS, both in AHS-endemic regions and during future incursions into currently AHSV-free areas. However, AHSV-LAV confers critical and effective protection for susceptible horses in AHS-endemic areas and, although potentially safer recombinant AHSV vaccines have proven effective in laboratory studies $(37,38)$, these are not available commercially and they are yet to be evaluated in the field. Until alternative vaccines become commercially available, control of AHS will remain reliant on the use of AHSV-LAV coupled with the adoption of strategies to minimize the likelihood of natural dissemination of revertant and reassortant vaccinederived viruses.

\section{Acknowledgments}

We thank the private-sector veterinarians, state veterinarians, and horse owners who collected samples and provided data used in this study.

Dr. Weyer is a veterinary research officer employed by the Equine Research Centre, Faculty of Veterinary Science, University of Pretoria, South Africa. She is authorized by the Western Cape provincial veterinary services to assist with equine movement control and disease surveillance within the province. Her areas of interest include African horse sickness epidemiology and other equine diseases, particularly those affecting the movement and trade of equines.

\section{References}

1. Zientara S, Weyer CT, Lecollinet S. African horse sickness. Rev Sci Tech. 2015;34:315-27. http://dx.doi.org/10.20506/rst.34.2.2359

2. Du Toit RM. Transmission of blue-tongue and horse-sickness by Culicoides. Onderstepoort J Vet Sci Anim Ind. 1944;19:7-16.

3. Maclachlan NJ, Guthrie AJ. Re-emergence of bluetongue, African horse sickness, and other orbivirus diseases. Vet Res. 2010;41:35. http://dx.doi.org/10.1051/vetres/2010007

4. Maclachlan NJ, Mayo CE. Potential strategies for control of bluetongue, a globally emerging, Culicoides-transmitted viral disease of ruminant livestock and wildlife. Antiviral Res. 2013;99:79-90. http://dx.doi.org/10.1016/j.antiviral.2013.04.021

5. Guichard S, Guis H, Tran A, Garros C, Balenghien T, Kriticos DJ. Worldwide niche and future potential distribution of Culicoides imicola, a major vector of bluetongue and African horse sickness viruses. PLoS One. 2014;9:e112491. http://dx.doi.org/10.1371/ journal.pone.0112491

6. Maclachlan NJ, Mayo CE, Daniels PW, Savini G, Zientara S, Gibbs EPJ. Bluetongue. Rev Sci Tech. 2015;34:329-40. http://dx.doi.org/10.20506/rst.34.2.2360

7. Guthrie AJ, Weyer CT. African horse sickness. In: Sprayberry KA, Robinson NE, editors. Robinson's current therapy in equine medicine. 7th ed. Philadelphia: Saunders; 2015. p. 150-1.

8. Theiler A. Die Sudafrikanische Pferdesterbe. Deuts Tierarztl Wschr. 1901;9:201-3, 221-6, 233-7, 241.

9. M'Fadyean J. African horse sickness. J Comp Pathol Ther. 1900;13:1-20. http://dx.doi.org/10.1016/S0368-1742(00)80001-6

10. von Teichman BF, Dungu B, Smit TK. In vivo cross-protection to African horse sickness serotypes 5 and 9 after vaccination with serotypes 8 and 6. Vaccine. 2010;28:6505-17. http://dx.doi.org/10.1016/j.vaccine.2010.06.105

11. World Organization for Animal Health. Infection with African horse sickness virus. Terrestrial Animal Health Code, 2015 [cited 2016 Jul 5]. http://www.oie.int/index.php?id=169\&L=0\&htmfile= chapitre_ahs.htm

12. Republic of South Africa Department of Agriculture, Forestry, and Fisheries. Animal Diseases Act, 1984 [cited 2016 Jul 5]. http://www.daff.gov.za/daffweb3/Branches/AgriculturalProduction-Health-Food-Safety/Animal-Health/importexport/ legislation/diseaseact

13. Sinclair M, Bührmann G, Gummow B. An epidemiological investigation of the African horsesickness outbreak in the Western Cape Province of South Africa in 2004 and its relevance to the current equine export protocol. J S Afr Vet Assoc. 2006;77:191-6. http://dx.doi.org/10.4102/jsava.v77i4.376

14. Quan M, van Vuuren M, Howell PG, Groenewald D, Guthrie AJ. Molecular epidemiology of the African horse sickness virus S10 gene. J Gen Virol. 2008;89:1159-68. http://dx.doi.org/10.1099/ vir.0.83502-0

15. Grewar JD, Weyer CT, Guthrie AJ, Koen P, Davey S, Quan M, et al. The 2011 outbreak of African horse sickness in the African horse sickness controlled area in South Africa. J S Afr Vet Assoc. 2013;84:1-7. http://dx.doi.org/10.4102/jsava.v84i1.973

16. Grewar JD. Suspected African horse sickness outbreakMelkbosstrand. Western Cape Government Epidemiology Report, 2013;5(5) [cited 2016 Jul 5]. http://www.elsenburg.com/vetepi/ epireport_pdf/May2013.pdf

17. Grewar JD. African horse sickness outbreak resolved. Western Cape Government Epidemiology Report, 2014;6(6) [cited 2016 Jul 5]. http://www.elsenburg.com/vetepi/epireport_pdf/June2014.pdf

18. World Organization for Animal Health. African horse sickness, South Africa [cited 2016 Jul 5]. http://www.oie.int/wahis_2/public/ wahid.php/Reviewreport/Review?page_refer=MapFullEventReport \&reportid $=5493$

19. World Organization for Animal Health. African horse sickness, South Africa [cited 2016 Jul 5]. http://www.oie.int/wahis_2/public/ 
wahid.php/Reviewreport/Review?page_refer=MapEventSummary \&reportid $=6650$

20. Guthrie AJ, Coetzee P, Martin DP, Lourens CW, Venter EH, Weyer CT, et al. Complete genome sequences of the three African horse sickness virus strains from a commercial trivalent live attenuated vaccine. Genome Announc. 2015;3:e0814-5. http://dx.doi.org 10.1128/genomeA.00814-15

21. Guthrie AJ, Coetzee P, Martin DP, Lourens CW, Venter EH, Weyer CT, et al. Complete genome sequences of the four African horse sickness virus strains from a commercial tetravalent live attenuated vaccine. Genome Announc. 2015;3:e1375-15. http://dx.doi.org 10.1128/genomeA.01375-15

22. Guthrie AJ, Maclachlan NJ, Joone C, Lourens CW, Weyer CT, Quan M, et al. Diagnostic accuracy of a duplex real-time reverse transcription quantitative PCR assay for detection of African horse sickness virus. J Virol Methods. 2013;189:30-5. http://dx.doi.org/10.1016/j.jviromet.2012.12.014

23. Weyer CT, Joone C, Lourens CW, Monyai MS, Koekemoer O, Grewar JD, et al. Development of three triplex real-time reverse transcription PCR assays for the qualitative molecular typing of the nine serotypes of African horse sickness virus. J Virol Methods. 2015;223:69-74. http://dx.doi.org/10.1016/j.jviromet.2015.07.015

24. Potgieter AC, Page NA, Liebenberg J, Wright IM, Landt O, van Dijk AA. Improved strategies for sequence-independent amplification and sequencing of viral double-stranded RNA genomes. J Gen Virol. 2009;90:1423-32. http://dx.doi.org/10.1099/ vir.0.009381-0

25. Kearse M, Moir R, Wilson A, Stones-Havas S, Cheung M, Sturrock S, et al. Geneious Basic: an integrated and extendable desktop software platform for the organization and analysis of sequence data. Bioinformatics. 2012;28:1647-9. http://dx.doi.org/10.1093/bioinformatics/bts199

26. Katoh K, Standley DM. MAFFT multiple sequence alignment software version 7: improvements in performance and usability. Mol Biol Evol. 2013;30:772-80. http://dx.doi.org/10.1093/molbev/ mst010

27. Guindon S, Dufayard JF, Lefort V, Anisimova M, Hordijk W, Gascuel O. New algorithms and methods to estimate maximumlikelihood phylogenies: assessing the performance of PhyML 3.0. Syst Biol. 2010;59:307-21. http://dx.doi.org/10.1093/sysbio/ syq010

28. Pommier T, Canbäck B, Lundberg P, Hagström A, Tunlid A. RAMI: a tool for identification and characterization of phylogenetic clusters in microbial communities. Bioinformatics. 2009;25:73642. http://dx.doi.org/10.1093/bioinformatics/btp051
29. Martin DP, Murrel B, Golden M, Khoosal A, Muhire B. RDP4: detection and analysis of recombination patterns in virus genomes. Virus Evol. 2015;1:1-5. http://dx.doi.org/10.1093/ve/vev003

30. Sugiyama K, Bishop DHL, Roy P. Analysis of the genomes of bluetongue viruses recovered from different states of the United States and at different times. Am J Epidemiol. 1982;115:332-47.

31. Nomikou K, Hughes J, Wash R, Kellam P, Breard E, Zientara S, et al. Widespread reassortment shapes the evolution and epidemiology of bluetongue virus following European invasion. PLoS Pathog. 2015;11:e1005056. http://dx.doi.org/10.1371/journal. ppat. 1005056

32. Weyer CT, Quan M, Joone C, Lourens CW, MacLachlan NJ, Guthrie AJ. African horse sickness in naturally infected, immunised horses. Equine Vet J. 2013;45:117-9. http://dx.doi.org/10.1111/ j.2042-3306.2012.00590.x

33. Martin LA, Meyer AJ, O'Hara RS, Fu H, Mellor PS, Knowles NJ, et al. Phylogenetic analysis of African horse sickness virus segment 10: sequence variation, virulence characteristics and cell exit. Arch Virol Suppl. 1998;14:281-93.

34. Huismans H, van Staden V, Fick WC, van Niekerk M, Meiring TL. A comparison of different orbivirus proteins that could affect virulence and pathogenesis. Vet Ital. 2004;40:417-25.

35. Potgieter AC, Wright IM, van Dijk AA. Consensus sequence of 27 African horse sickness virus genomes from viruses collected over a 76-year period (1933 to 2009). Genome Announc. 2015;3:e0092115. http://dx.doi.org/10.1128/genomeA.00921-15

36. Nevill EM, Venter GJ, Edwardes M, Pajor ITP, Meiswinkel R, van Gas JH. Culicoides species associated with livestock in the Stellenbosch area of the Western Cape Province, Republic of South Africa (Diptera: Ceratopogonidae). Onderstepoort J Vet Res. 1988;55:101-6.

37. Guthrie AJ, Quan M, Lourens CW, Audonnet JC, Minke JM, Yao J, et al. Protective immunization of horses with a recombinant canarypox virus vectored vaccine co-expressing genes encoding the outer capsid proteins of African horse sickness virus. Vaccine. 2009;27:4434-8. http://dx.doi.org/10.1016/j.vaccine.2009.05.044

38. Alberca B, Bachanek-Bankowska K, Cabana M, Calvo-Pinilla E, Viaplana E, Frost L, et al. Vaccination of horses with a recombinant modified vaccinia Ankara virus (MVA) expressing African horse sickness (AHS) virus major capsid protein VP2 provides complete clinical protection against challenge. Vaccine. 2014;32:3670-4. http://dx.doi.org/10.1016/j.vaccine.2014.04.036

Address for correspondence: Alan J. Guthrie, University of Pretoria, Equine Research Centre, Faculty of Veterinary Science, Pretoria 0002, South Africa; email: alan.guthrie@up.ac.za

\section{The Public Health Image Library (PHIL)}

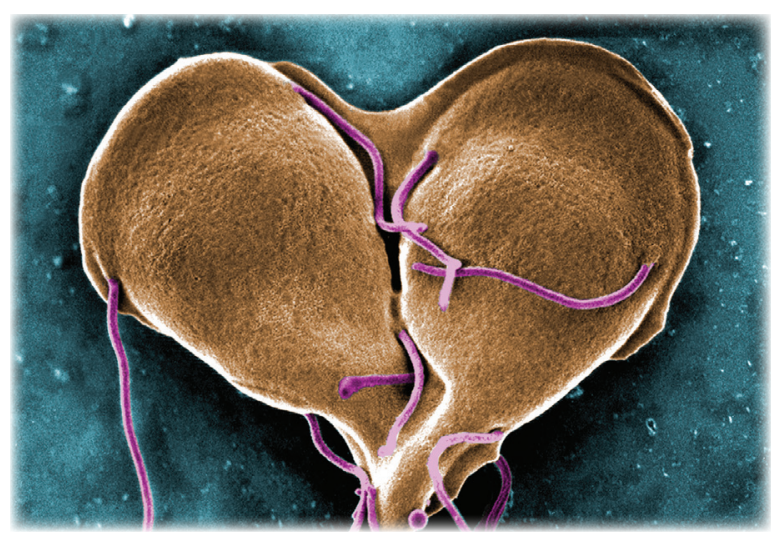

The Public Health Image Library (PHIL), Centers for Disease Control and Prevention, contains thousands of public health-related images, including high-resolution (print quality) photographs, illustrations, and videos.

PHIL collections illustrate current events and articles, supply visual content for health promotion brochures, document the effects of disease, and enhance instructional media.

PHIL images, accessible to PC and Macintosh users, are in the public domain and available without charge.

Visit PHIL at http://phil.cdc.gov/phil 